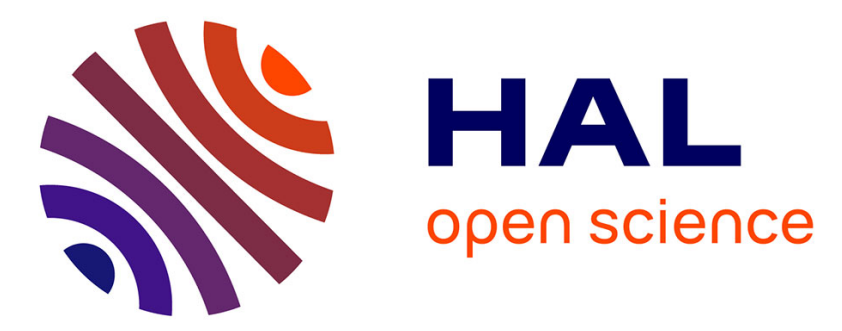

\title{
Processus de régionalisation des productions matérielles et des modes d'acquisition de modèles exogènes en Corse à l'âge du Bronze et au premier âge du Fer
} Kewin Peche-Quilichini

\section{- To cite this version:}

Kewin Peche-Quilichini. Processus de régionalisation des productions matérielles et des modes d'acquisition de modèles exogènes en Corse à l'âge du Bronze et au premier âge du Fer. Archimède: archéologie et histoire ancienne, 2014, 1, pp.191-202. halshs-01586640

\section{HAL Id: halshs-01586640 \\ https://shs.hal.science/halshs-01586640}

Submitted on 13 Sep 2017

HAL is a multi-disciplinary open access archive for the deposit and dissemination of scientific research documents, whether they are published or not. The documents may come from teaching and research institutions in France or abroad, or from public or private research centers.
L'archive ouverte pluridisciplinaire HAL, est destinée au dépôt et à la diffusion de documents scientifiques de niveau recherche, publiés ou non, émanant des établissements d'enseignement et de recherche français ou étrangers, des laboratoires publics ou privés. 


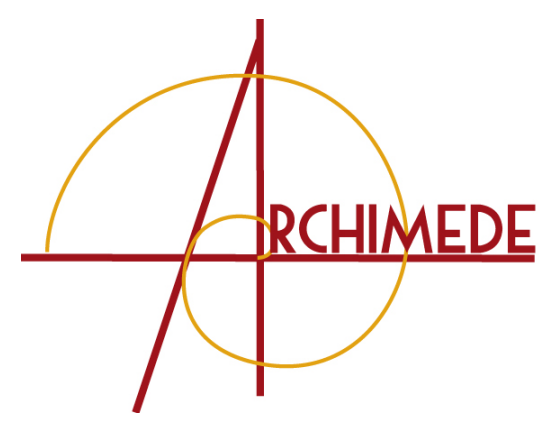

\section{PROCESSUS DE RÉGIONALISATION DES PRODUCTIONS MATÉRIELLES ET DES MODES D'ACQUISITION DE MODĖLES EXOGĖNES EN CORSE À L'ÂGE DU BRONZE ET AU PREMIER ÂGE DU FER}

\section{Kewin PECHE-QUILICHINI}

Chercheur associé, LAMPEA UMR 7269, Aix-Marseille Université. ASM-Archéologie des Sociétés Méditerranéennes, UMR5140, Univ. Montpellier 3, CNRS, MCC, 34000 Montpellier, France. Labex ARCHIMĖDE, programme «Investissement d'Avenir »

ANR-11-LABX-0032-01

\section{RÉSUMÉ}

bainzu.di.baiucheddu@voila.fr

L'analyse globale et croisée des séquences matérielles protohistoriques en provenance de Corse permet de proposer des cadres techniques, typologiques, stylistiques, chronologiques et, dans certains cas, fonctionnels. En outre, la contextualisation du corpus ici étudié et sa confrontation avec les données des régions voisines aboutissent à une reconstitution esquissée des interactions et évitements entre les groupes de producteurs-consommateurs considérés dans l'espace tyrrhénien. À l'échelle de l'île, il est ainsi possible d'observer une partition diachronique et bipolaire des groupes techno-stylistiques, entre la partie nord-est et l'espace méridional. L'autonomie de ces deux ensembles s'exprime depuis le Bronze ancien jusqu'à une phase avancée du second âge du Fer. L'examen de ce phénomène permet de retracer la géographie culturelle complexe de ces sociétés insulaires, que I'on suppose en partie liée aux traits du relief géographique et à l'étendue de l'aire d'influence des contextes culturels voisins. La Corse apparaît, en effet, comme un ensemble récepteur, même si la situation locale est hétérogène. L'étude des sphères productives de Sardaigne et d'Italie tyrrhénienne permet d'estimer l'ampleur, les formes, la direction et les spécificités microrégionales des mécanismes d'emprunt entre les aires ici présentées. Les échanges techniques et morphologiques viennent ainsi

Mots-CLÉs

Corse,

âge du Bronze,

âge du Fer,

transfert,

géographie culturelle. documenter les dynamiques de contact et les autres processus d'interaction entre groupes humains séparés par des bras de mer aisément franchissables aux époques protohistoriques.
The comprehensive and comparative analysis of the Protohistorical material sequences from Corsica allows to propose some technical, typological, stylistic, chronological and, in some case, functional frameworks. Moreover, the contextualization of the studied corpus and his confrontation with data from the neighboring regions lead to a drafted reconstruction of interactions and avoidances between the considered groups of producers and consumers in the Tyrrhenian area. On the scale of the island, it is possible to observe a diachronic and bipolar partition of techno-stylistic groups, between the Northeastern and Southern parts. The autonomy of these two zones is manifested from the Early Bronze Age to an advanced stage of the Late Iron Age. The examination of that phenomenon allows to trace the complex cultural geography of these insular societies, which is supposedly due, at least in partly, to the landscape shape and to the extent of the area of influence of neighboring cultural contexts. Corsica appears indeed as a receptor zone, even if the local situation is heterogeneous. The study of Sardinian and Italo-Tyrrhenian productive spheres enables to estimate the extent, the forms, the directions and the micro-regional specificities of influence mechanisms between the considered areas. The technical and morphological exchanges document the contact dynamics and the other socio-cultural process of interaction between human groups separated by easily crossable sea inlets during Protohistorical times.
KeYwords 


\section{INTRODUCTION}

Cette contribution traite de l'intégralité de la Protohistoire de la Corse au travers de quelques exemples précis illustrant certains phénomènes culturels faisant intervenir des processus d'interaction. Comme on le verra par la suite pour bien des cas, I'analyse multi-thématique des dynamiques met en évidence une grande complexité de ces mécanismes culturels, avec des formes de superposition, de prolongation, d'évitement, d'isolement, de métissage, réciproque ou non, de transferts spécifiques, etc., qui peuvent elles-mêmes être interprétées de manières très diverses. Ces réflexions sont tirées de nos recherches universitaires sur les vaisselles céramiques protohistoriques de Corse [1] et de travaux de terrain [2], notamment sur les différents aspects de la transition Bronze/Fer dans le sud de l'île.

\section{INTERACTION, TRANSFERT ET INSULARITÉ : QUELQUES GENEERALITÉS THÉORIQUES}

Avant de proposer des exemples concrets, il nous semble opportun de poser les bases théoriques de la notion d'interaction, et notamment de ses causes, en rappelant que l'action consciente ou inconsciente d'un groupe sur un autre se fait obligatoirement par l'intermédiaire d'un contact entre les personnes, que ce contact peut induire d'autres formes d'échange, comme celui des matériaux, des objets finis, des modèles technico-morpho-stylistiques et des idées, et que, dans le cas de la Corse, ces transferts horizontaux ne peuvent se faire que par voie maritime. Dans le cas de transferts verticaux, c'est la tradition qui est vecteur d'interaction entre un groupe, sa propension à innover et les barrières véhiculées par ses savoir-faire et ses vouloir-faire vernaculaires. Les contextes insulaires, à plus forte raison lorsqu'ils sont montagneux, ont souvent été perçus comme des « niches » ou des « refuges » culturels, propices à la conservation de traditions sur des temps plus dilatés qu'en des zones plus facilement et/ou plus directement accessibles et fréquentées par des éléments exogènes. On tentera ici de vérifier ce point.

À l'autre extrémité de ce postulat, dans la plupart des cas, l'archéologie ne permet d'observer que la conséquence des dynamiques de contact, en relevant la présence d'éléments intrusifs et autres «moutons noirs » dans des séquences qui ne sont référentes que parce qu'elles constituent des instantanés (fig. 1). Autrement dit, il est probable qu'un nombre non négligeable d'interactions culturelles nous échappe car ne contrastant pas assez avec le noyau supposé dur d'ensembles que nous

[1] PeCHE-QUilichini à paraître 1.

[2] Milletti et al. 2012. pensons cohérents. On précisera cet aspect par un appel constant aux données fournies par l'archéologie italienne, insulaire et continentale, seule aire géographique offrant une cohérence culturelle à la contextualisation des informations apportées par les travaux menés en Corse.

\section{LA CORSE : UN CONTEXTE INSULAIRE}

On initiera ce développement par un bref rappel géographique, primordial dans l'optique de contextualiser les raisonnements qui vont suivre. La Corse est la grande île la plus septentrionale de Méditerranée, avec une superficie d'environ $9000 \mathrm{~km}^{2}$, ce qui la classe au quatrième rang derrière Chypre. Elle est distante de $13 \mathrm{~km}$ de la Sardaigne au sud, de $80 \mathrm{~km}$ de la Toscane continentale (à $40 \mathrm{~km}$ de l'île d'Elbe) à l'est et de 180 km de la Côte d'Azur au nord. $90 \%$ du territoire est occupé par des massifs montagneux rythmés par une centaine de sommets dépassant les 2000 m et culminant à 2706 m au Monte Cintu.

Figure 1 : schéma théorique d'interactivité entre trois zones voisines selon deux évolutions possibles (échanges restreints et échanges nombreux et généralisés).

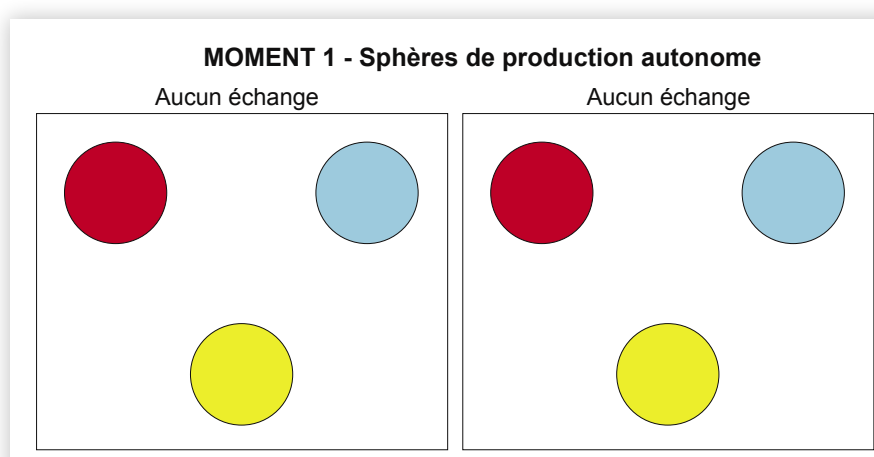

MOMENT 2 - Dynamiques de contact Système d'échanges élémentaires restreint Système d'échanges généralisés
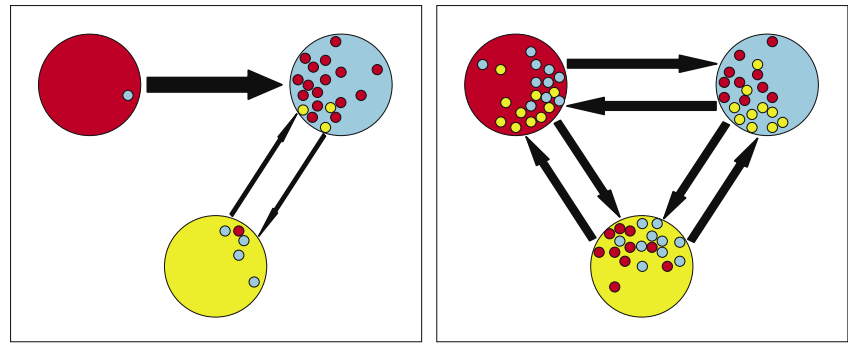

MOMENT 3 - Reformation des sphères de production par métissage Métissage restreint Fort métissage

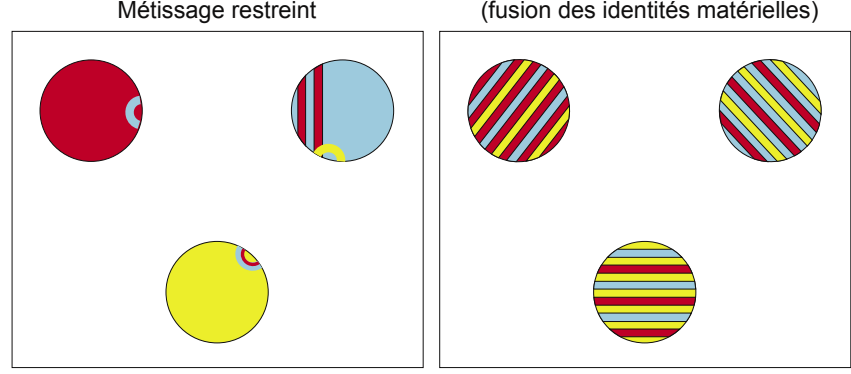


Ce relief a engendré un important découpage du littoral et permet à la Corse de disposer d'une façade maritime longue de plus de $1000 \mathrm{~km}$, soit plus que le littoral méditerranéen français entre Menton et Collioure. La côte présente généralement un aspect assez contrasté, formé de golfes, de presqu'îles et d'écueils, à l'exception d'une frange orientale de près d'une centaine de kilomètres, qui est rectiligne et continue (entre Solenzara et Bastia). Cette situation, conjuguée à un climat rude en montagne, a engendré plusieurs formes de cloisonnement, végétal, animal ou humain, et ce, jusqu'à des époques récentes. L'évidence, voire l'omniprésence de la montagne dans ce contexte insulaire, a très souvent, dans I'histoire, engendré une partition de l'île en deux voire trois ensembles culturels et/ou politiques, avec d'un côté une Corse tournée vers I'Italie, et de l'autre, une Corse tournée vers la Sardaigne et vers l'ouest, en fonction des co-visibilités. Le régime actuel de bidépartementalisation de l'île obéit d'ailleurs à un découpage d'époque médiévale calqué sur une bipolarité articulée par la principale ligne de partage des eaux. On remarquera d'ailleurs avec intérêt que les évidences culturelles fournies par l'archéologie protohistorique montrent, à quelques exceptions près, que la direction des cours d'eau indique souvent le pôle dynamique externe qui bénéficie de la plus profonde infiltration au sein du bassin versant concerné. À ce titre, on pourrait mentionner la circulation des fibules villanoviennes jusqu'au cœur du Niolu (vallée du Golu orientée vers I'Étrurie septentrionale), la présence de mobiliers nettement influencés par le Bronze moyen latial sur les sites de Mar'e Stagnu et Sant'Agata (vallée du Tavignanu orientée vers l'Étrurie méridionale) [3], la présence de boutons nuragiques dans le sud du Fium'Orbu (vallée du Travu orientée vers la Gallura) ou de modèles architecturaux proches de modèles sardes dans tout le sud de l'île (vallées du Stabiacciu, du Tàravu, du Rizzanese, de la Nivara, de l'Ortolu orientées vers la Sardaigne). Dans le relief contrasté de lî̂le, les vallées fluviatiles constituent des voies de pénétration naturelles utilisées par les hommes et donc par leurs idées.

\section{L'ÂGE DU BRONZE : DES CULTURES MATÉRIELLES ET DES ARCHITECTURES SOUS PERFUSION EXTERNE}

On abordera ici le thème des occurrences constatées dans les sphères productives et architecturales entre l'expression insulaire et celle de ses plus proches voisins, afin de proposer une géographie culturelle du dynamisme et du rayonnement des groupes tyrrhéniens tout au long de la Protohistoire.

Sur l'île, l'architecture caractéristique du II $^{\mathrm{e}}$ millénaire est dominée par le modèle de la torra [4]. Les torre sont des monuments en forme de tour tronconique, élevés en gros blocs, au nombre d'une soixantaine dans l'île, et qui constituaient des greniers collectifs fortifiés en même temps probablement qu'un élément ostentatoire des communautés de l'âge du Bronze ancien puis moyen (fig. 2).

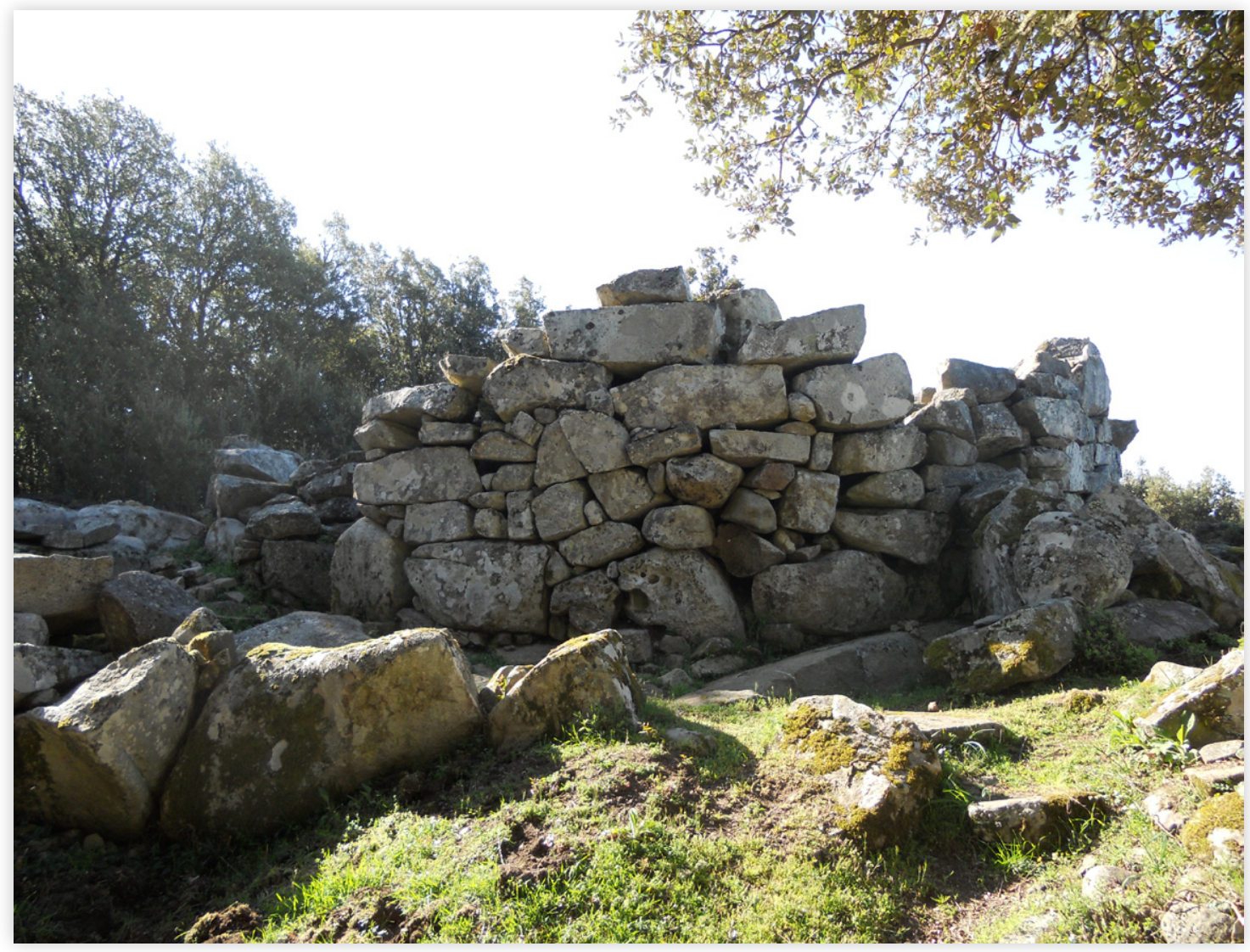

Figure 2

vue de la torra de Tusiu (photo de l'auteur).

[3] ROTH \& CONGÈS 1976.

[4] PeChe-Quilichini 2011 
Figure 3 : distribution géographique des torre et mise en évidence des deux zones de concentrations (lignes pointillées). Les trois monuments du nord de l'île sont douteux.

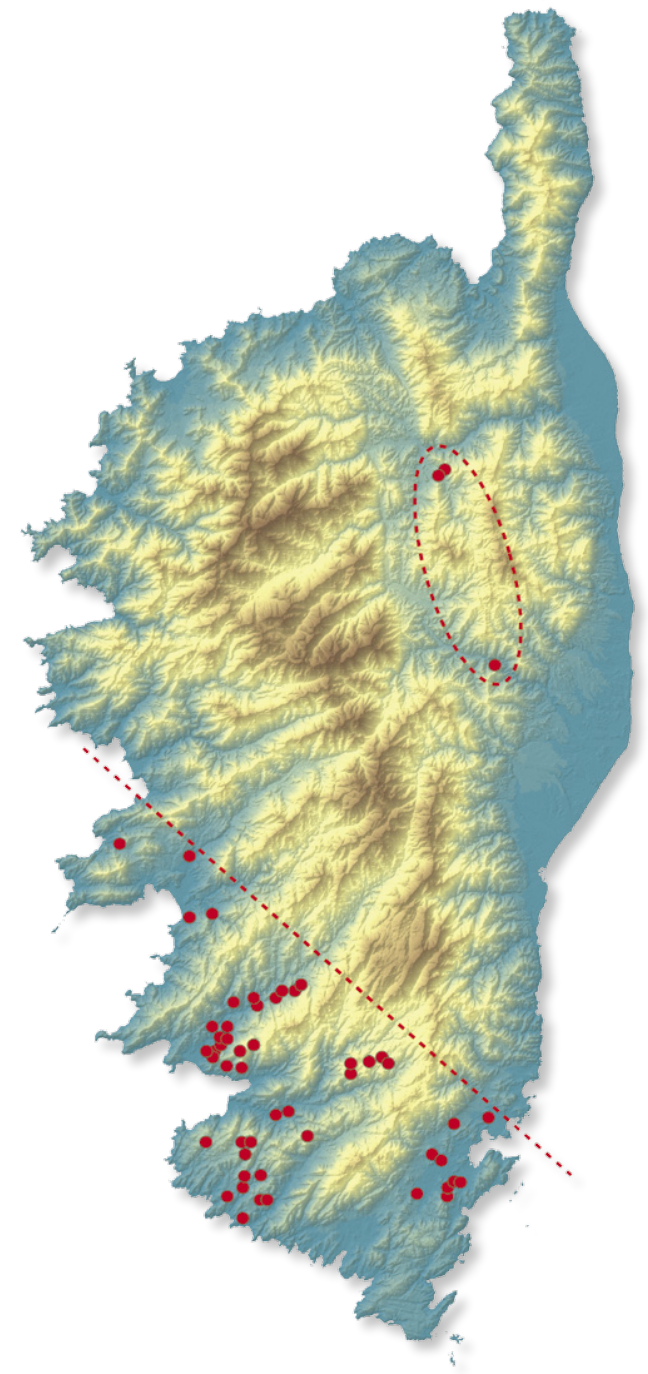

Il s'agit de constructions complexes, à superposition de voûtes, dont la distribution préférentielle dans le sud de l'île (fig. 3) s'accorde bien avec l'origine sarde du modèle architectural et fonctionnel qu'est le nuraghe. Les nuraghi constituent le cœur économique et ostentatoire des communautés nuragiques au Bronze ancien et moyen. La Sardaigne en compterait près de 7000. Parallèlement, l'infiltration culturelle sarde est également perceptible dans les formes et les fonctions de la vaisselle avec la diffusion des coupes

Figure 5 : hache du Bronze ancien, alliage base cuivre, Mignataghja (photo : musée départemental d'Aleria).

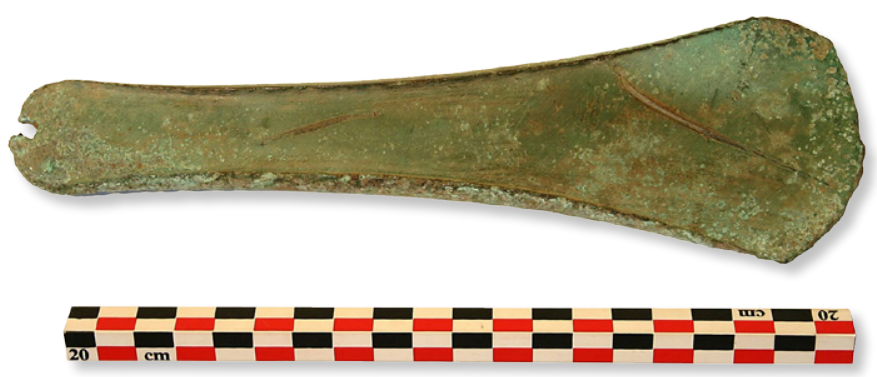

Figure 4 : en noir, part du mobilier présentant des affinités sardes dans les séquences céramiques du Bronze ancien.

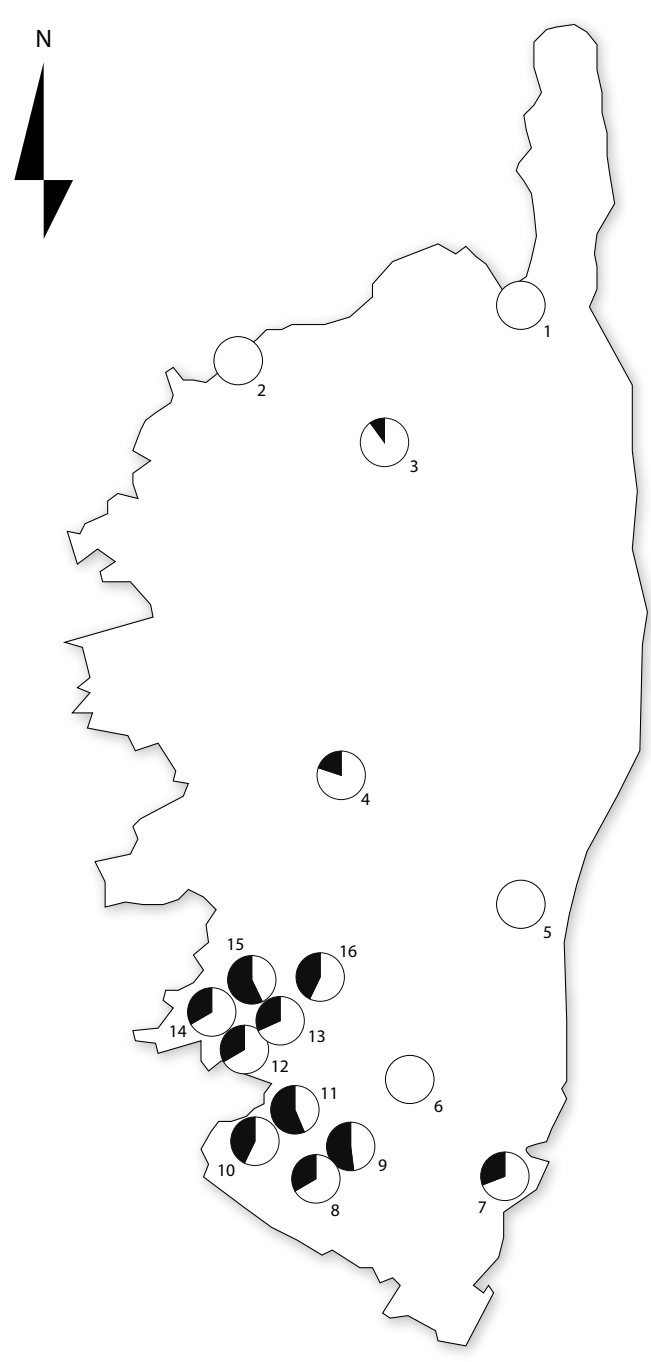

à pied haut et des tasses à anse coudée, essentiellement dans les sépultures [5] du sud de la Corse. La quantification des formes sardes dans les ensembles de vaisselle de la fin du Bronze ancien montre qu'il existe un gradient géographique marqué par une diminution progressive de I'influence sarde (fig. 4) si I'on se déplace vers le nord [6]. Par ailleurs, même si cela est moins spectaculaire, il faut noter la reconnaissance ponctuelle d'éléments typologiques d'origine liguro-provençale, comme certaines tasses à carène basse, ou de formes directement inspirées de répertoires toscans. Cet impact de l'Italie centro-occidentale est également perceptible dans certains objets métalliques (comme par exemple la hache de type Burzanella provenant de Mignataghja ; fig. 5), pour l'heure impossibles à caractériser : imitations ou importations, que I'on retrouve de préférence sur la côte orientale de l'île [7].

[5] PECHE-Quilichini à paraître 2.

[6] PeCHE-Quilichini à paraître 3.

[7] PeCHE-Quilichini à paraître 4. 
Du point de vue symbolico-fonctionnel, on remarquera que les contextes sépulcraux intègrent généralement plus d'éléments exogènes que les ensembles domestiques, alors même que les pratiques funéraires s'inscrivent pleinement dans une tradition millénaire.

Au Bronze moyen, force est de constater que la Corse est toujours (et plus profondément) touchée par le dynamisme des groupes toscans (faciès de Grotta Nuova) et que ces mécanismes sont particulièrement évidents à la lecture des grilles de morphologie céramique, avec par exemple les sites de Monte Ortu et de Filitosa [8]. Quelques cas particuliers montrent aussi une infiltration de modèles issus du répertoire piémontais du style de Viverone. L'examen de la carte de répartition de ces ensembles (fig. 6), qui sont d'abord imités avant d'être réinterprétés, montre que les sites concernés ont une

Figure 6 :

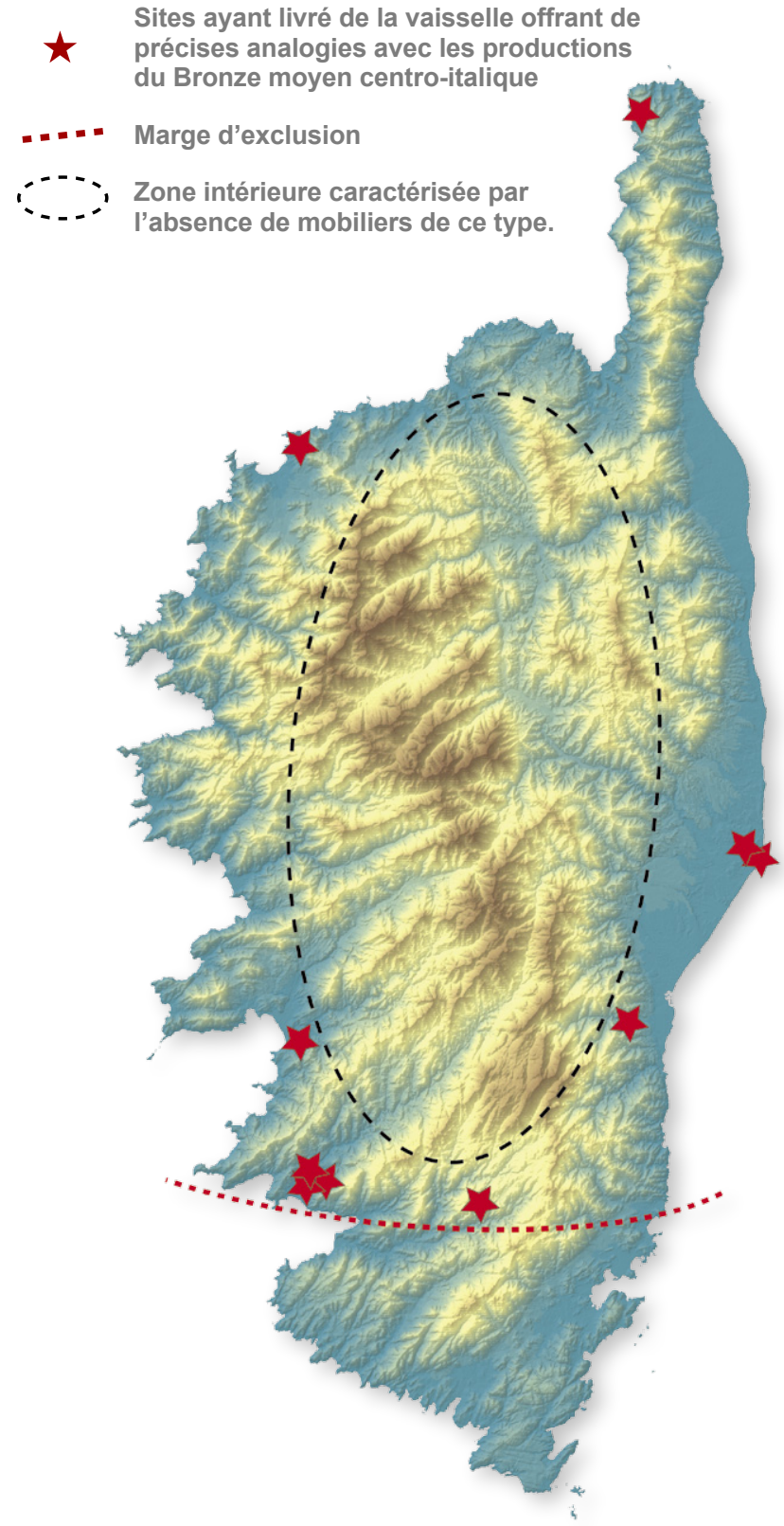

Figure 7 : répartition des sites ayant livré des fonds aplatis sur vannerie en Sardaigne.

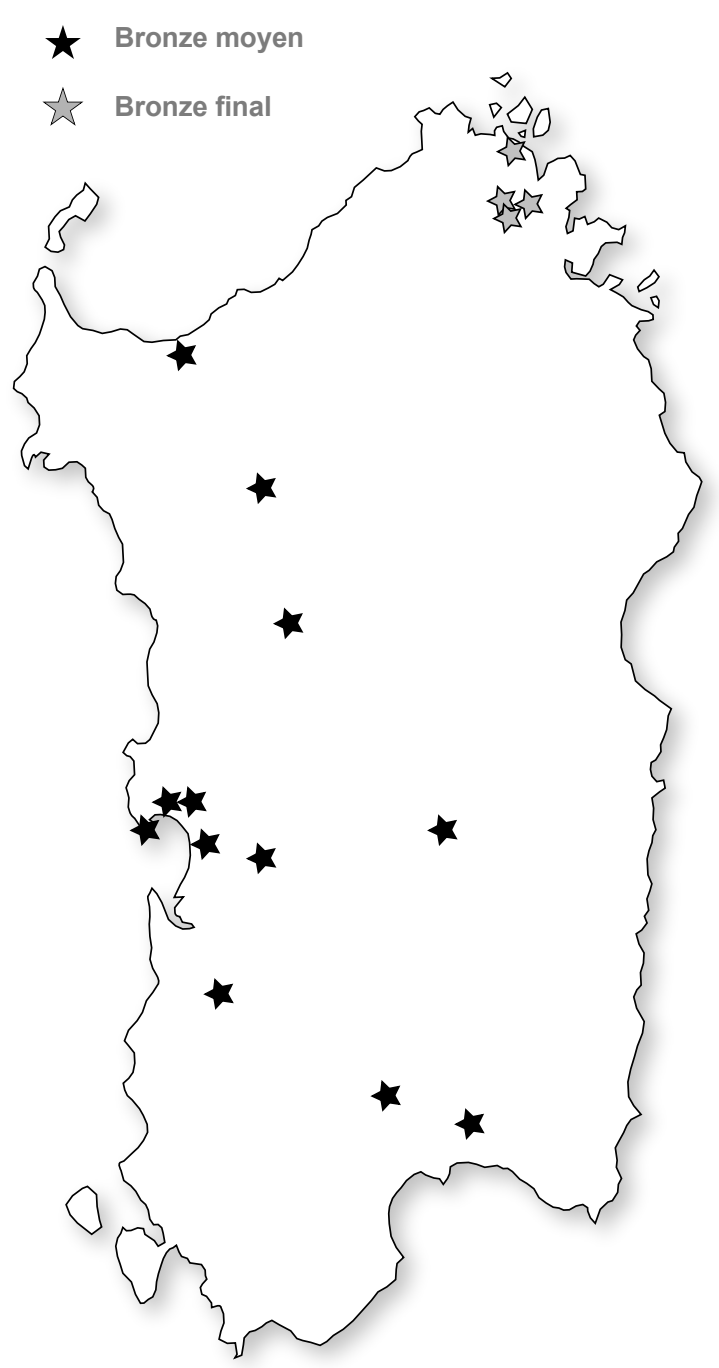

distribution plutôt littorale dont la dispersion géographique ne touche que peu ou pas les sites du sud de l'île (Sartenais et région de Porto-Vecchio). Autrement dit, les vaisselles de filiation toscane du début du Bronze moyen sont absentes des sites d'ascendance architecturale sarde des vallées méridionales alors même que c'est dans ce secteur que les contextes sont le mieux documentés, ce qui traduit probablement une volonté bien consciente. Durant la seconde moitié de la période, phase la moins documentée de la Protohistoire insulaire, la propension à l'externalisation des initiatives italiques semble bien moindre. Ce basculement des dynamiques est également perceptible en d'autres régions de Méditerranée occidentale (Lachenal 2008 ; Vital 1998). Au Bronze final, la distinction des régions Sud se renforce avec l'émergence rapide d'un groupe culturel homogène baptisé « faciès Apazzu-Castidetta-Cucuruzzu » [9].

[8] Atzeni 1966 ; Atzeni \& Depalmas 2006 ; Lachenal \& Peche-Quilichini 2009 ; PeChe-Quilichini 2009b ; PeChe-Quilichini à paraître 2.

[9] PeChe-Quilichini 2010. 
Figure 8 : mise en évidence des connexions culturelles inhérentes à la présence de quatre traceurs caractéristiques de la production céramique du début du Bronze final dans le sud de la Corse.

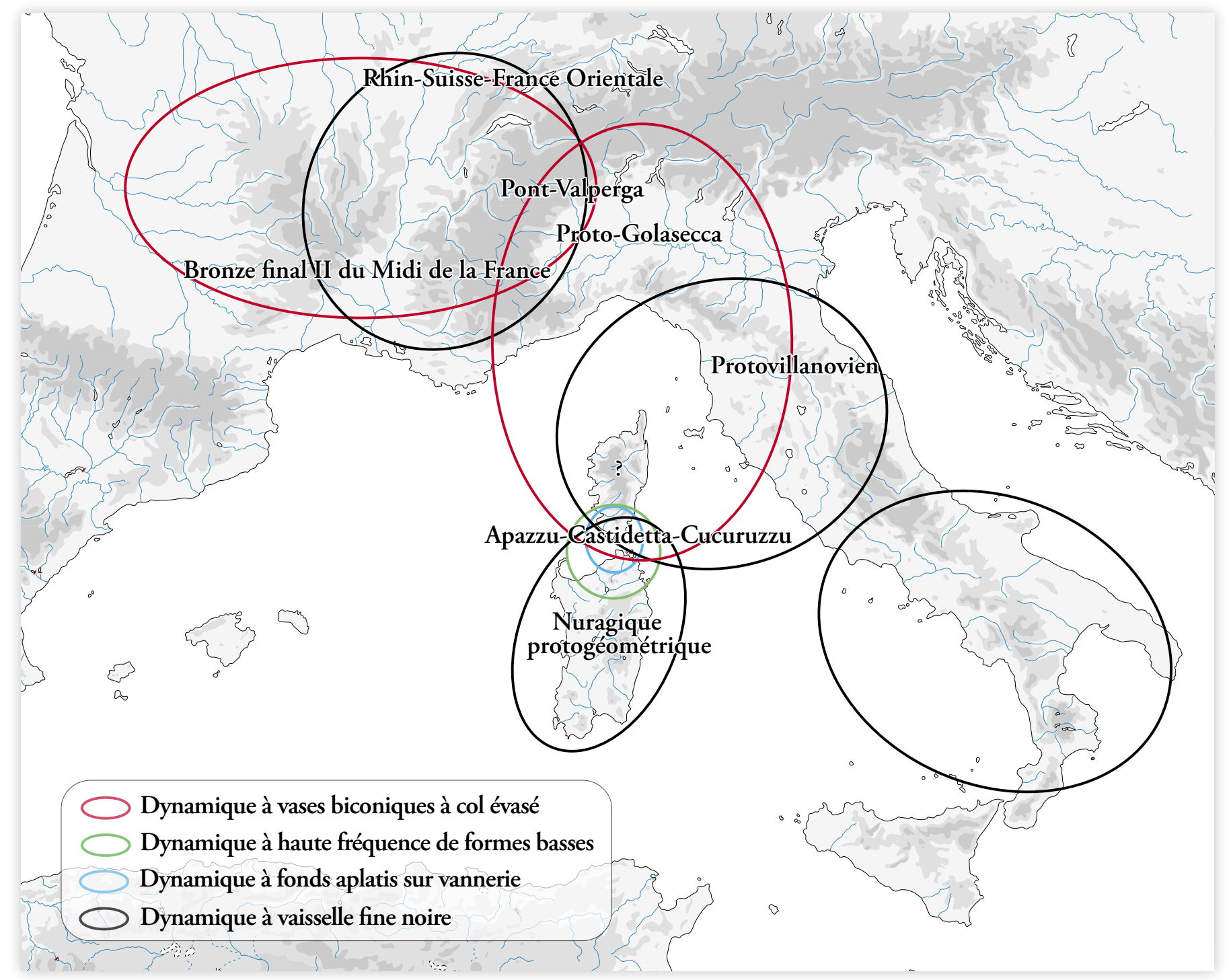

Dans le même temps, les groupes du centre et du nord de l'île nous sont totalement inconnus, très certainement à cause d'une industrie que I'on ne sait pas à ce jour individualiser. Il s'agirait donc en l'état d'un problème de reconnaissance. Dans le sud, le faciès «A.C.C. » est bien identifié, notamment grâce à une production céramique stéréotypée, au sein de laquelle il faut mentionner l'importante fréquence de formes biconiques présentant un rebord évasé. Sans entrer dans les détails, il est possible d'évoquer la diffusion de cette forme dans lîle à partir de contextes nord-italiques, ligures ou toscans. L'autre forme caractéristique de l'époque est l'assiette de forme simple, munie ou pas d'un couple d'anses rubanées, et qui sert souvent de couvercle. Il s'agit là de vaisselles qui trouvent d'étroites correspondances, en termes de morphologie comme de fréquence, avec les séquences des habitats du Bronze final du nord de la Sardaigne. Cette vaisselle corse méridionale du Bronze final est également caractérisée par une dichotomie de la production avec d'un côté près de $85 \%$ de céramique « commune » et, de l'autre, $15 \%$ de céramique « fine ». Dans ce cadre, on observe que les chaînes opératoires ne sont pas ramifiées et que la distinction se fait dès le moment du choix des matériaux et jusqu'à la typologie et probablement jusqu'aux aspects fonctionnels. Cette partition de la production domestique de la poterie trouve un écho dans de nombreux contextes voisins, Toscane, Italie du Sud, Sardaigne, vallée du Rhône, entre autres. Enfin, un autre traceur du Bronze final est la fréquence de la technique d'aplatissement des fonds par percussion posés sur vannerie, technique qui touche essentiellement les formes basses [10]. Il s'agit d'un procédé visant la standardisation des gabarits, notamment ceux des couvercles, et qui fait intervenir des vanneries discoïdes à tressage concentrique. Cette technique arrive indéniablement de Sardaigne (fig. 7), où ces protocoles sont déjà présents au Bronze moyen [11].

[10] PeChe-Quilichini 2009a.

[11] SeBIS 1995. 


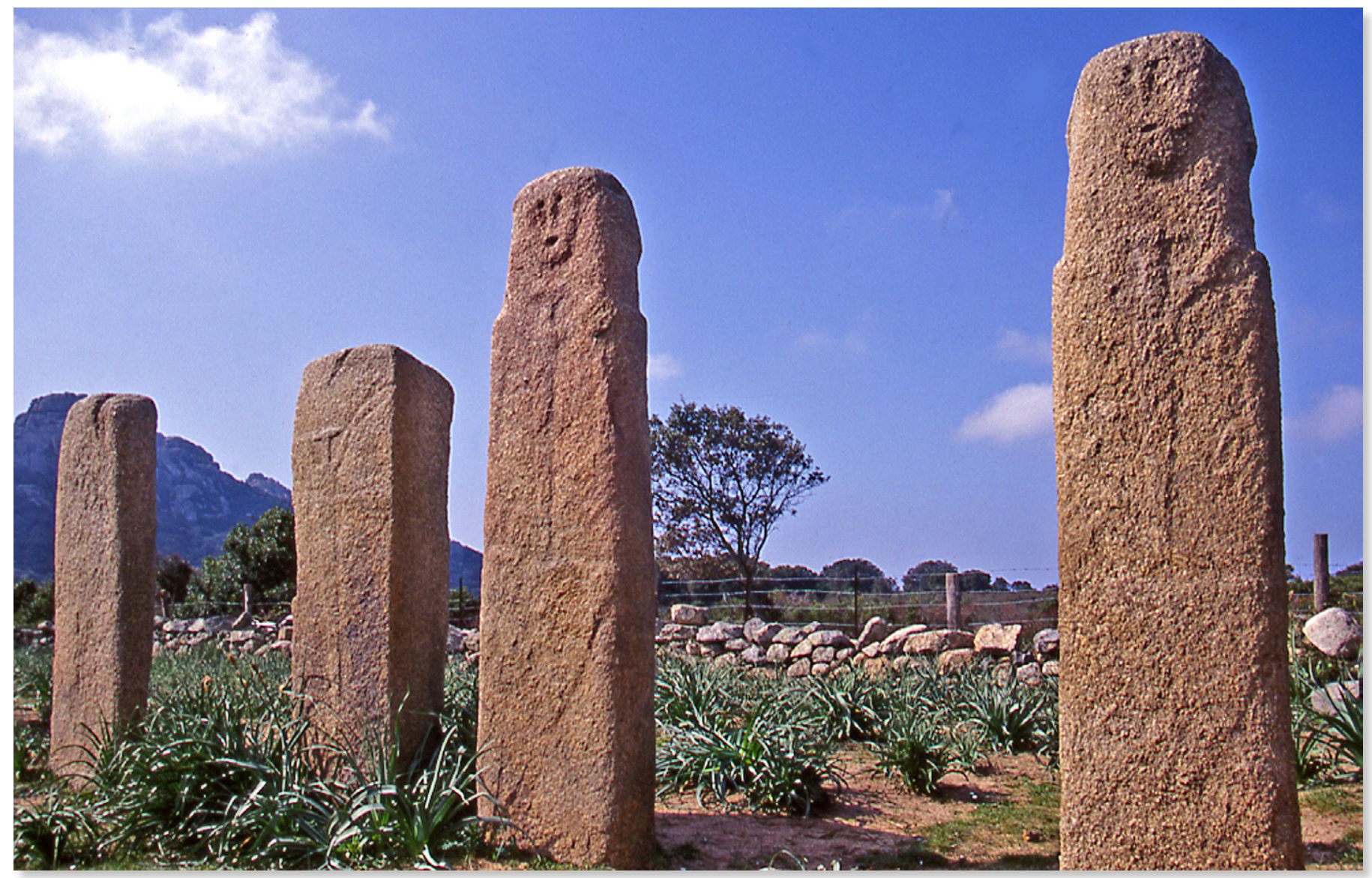

Au final, pour cette période et au moins pour la production potière, on peut conclure à une constitution assez métissée des assemblages (fig. 8), selon un axe privilégié qui lie le nord de la Sardaigne au sud de la Corse et qui, selon certains travaux ethnographiques récents [12], pourrait trahir la pratique d'alliances exogamiques entre ces deux régions séparées par la mer mais en situation de co-visibilité. Notons encore que ce schéma pourrait tout aussi bien être appliqué aux industries métalliques, puisque la Corse connaît, à partir du $\mathrm{XII}^{\mathrm{e}}$ siècle, un net développement technologique qui concerne le travail du bronze, avec notamment l'introduction de modèles sardes, comme par exemple les haches et ciseaux « à marges rehaussées » (exemplaires de Torre et du Monti Barbatu, moule de Punta Ficaghjola) [13]. La répartition des statues-menhirs portant une arme dans le sud de I'île atteste une autre forme de superposition territoriale [14]. En Corse, au Bronze final, la statuaire mégalithique anthropomorphe (fig. 9) connaît en effet un véritable essor qui touche l'intégralité de l'île. Cependant,

[12] Gosselain 2002.

[13] PECHE-QUILICHINi et al. à paraître.

[14] LEANDRI \& PECHE-QUILICHINI à paraître.

[15] D'Anna et al. 2006 ; D'Anna et al. 2007.

[16] PeCHe-Quilichini à paraître 1. c'est uniquement dans le sud, de façon contemporaine et spatialement parallèle (fig. 10) aux mécanismes de métissage observés pour les cultures matérielles, que les statues-menhirs vont se multiplier et se doter d'épées, de poignards et d'arcs, comme pour réaffirmer un ancrage territorial [15] et identitaire « menacé » par les infiltrations culturelles externes [16].

Figure 10 : distribution géographique comparée des statues-menhirs armées et des habitats du Bronze final.

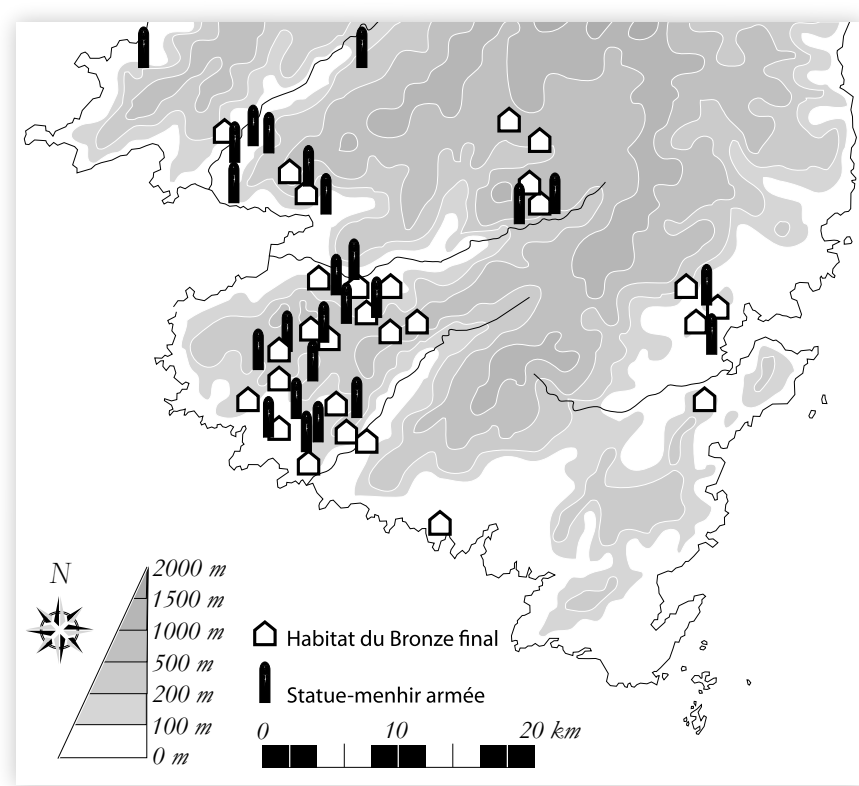




\section{L'ÂGE DU FER : AUTONOMIE DES SPHĖRES PRODUCTIVES}

Au premier âge du Fer, période pour laquelle on dispose de données pour l'ensemble des microrégions de l'île, sur le plan des assemblages céramiques, on a récemment pu définir trois à quatre groupes distincts et autonomes (fig. 11), incluant deux faciès principaux : le groupe de Nuciaresa dans le sud et le groupe de Tuani-Mizane dans le nord et le centre [17]. Au-delà des différences exprimées par les vaisselles, on a pu constater des disparités sur d'autres plans, avec notamment un grand nombre de mobiliers villanoviens (essentiellement des fibules) importés ou imités dans le nord alors que ces objets sont presque absents dans le sud [18], trahissant des disparités dans le

Figure 11 : géographie des faciès culturels du premier âge du Fer de Corse.

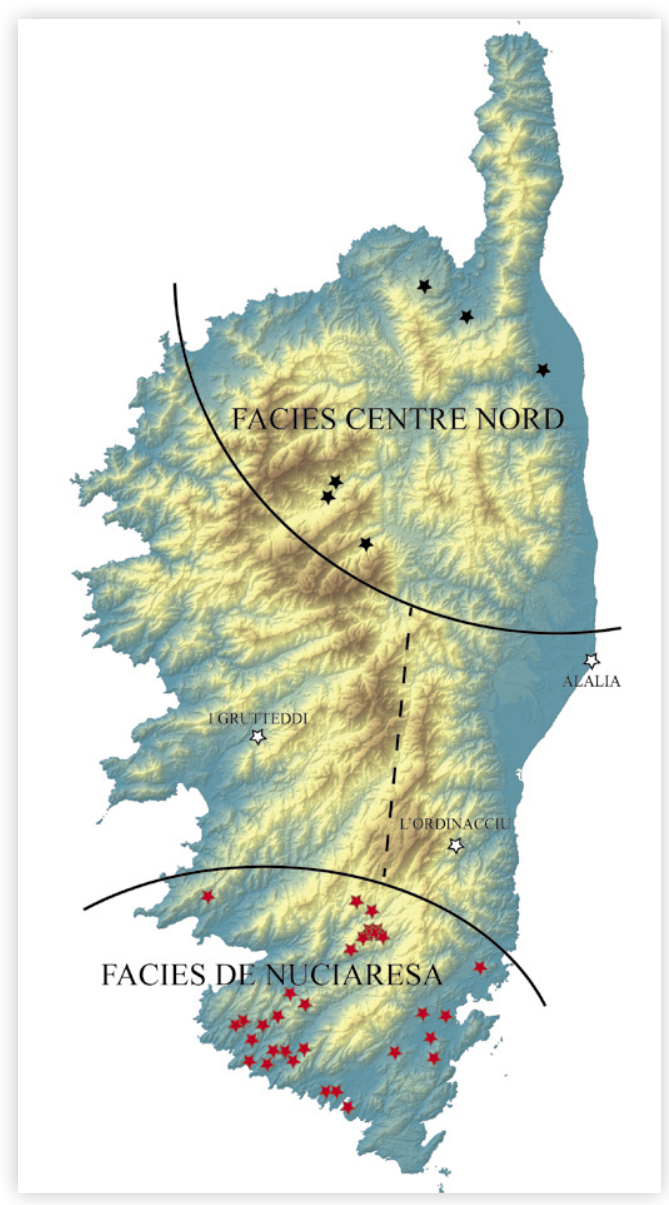

[17] PeChe-Quilichini à paraître 1 .

[18] LeChenAult 2011.

[19] Le calcul de l'indice de Jaccard (ij) permet de quantifier le rapport de similarité entre deux assemblages. La formule en est la suivante : $i j=n(a \beta) / n a+n \beta-n(a \beta) . n a=$ nombre de formes présentes dans I'assemblage $a ; n \beta=$ nombre de formes présentes dans l'ensemble $\beta$; $n(a \beta)=$ nombre de formes communes aux assemblages $a$ et $\beta$. Avec cette formule, l'indice sera toujours inférieur à 1 . Un indice fort indique un important degré de ressemblance entre deux assemblages. L'indice est ici calculé sur le nombre de types et non sur le nombre réel de récipients. Il s'agit donc d'un rapport calculé « à la baisse ».

[20] PECHE-QUILICHINI à paraître 1 . mode d'acquisition des denrées extérieures et/ou dans les codes vestimentaires. Les vallées méridionales semblent fonctionner en totale autarcie culturelle et économique, à quelques exceptions près sur lesquelles nous reviendrons. Concernant uniquement la production céramique, des travaux récents ont proposé une quantification du taux de ressemblance/dissemblance entre les contextes du nord et du sud au moyen du calcul de l'indice de Jaccard, qui permet de chiffrer les superpositions typologiques, $y$ compris pour des séries quantitativement hétérogènes [19] En règle générale, on considère que pour les époques protohistoriques et les collections céramiques modelées, un indice supérieur à 0,3 traduit un taux de superposition typologique significatif. Ici, I'indice (fig. 12 et $\mathbf{1 3}$ ) montre que la filiation entre les industries du Bronze final et du premier âge du Fer dans le sud est forte alors que les assemblages contemporains du nord et du sud sont nettement discordants [20]. Autrement dit, les mécanismes de perduration en diachronie des styles et des techniques sont en Corse plus forts que les tendances transgressives en synchronie. Ce rapport entre tradition et transgression illustre probablement assez bien un cloisonnement dicté par des géographies culturelles complexes et qui se traduit également dans d'autres sphères. Il en est par exemple ainsi dans le domaine de l'organisation de l'habitat.

Figure 12 : tableau croisé des mesures de l'indice de Jaccard entre les trois principaux faciès céramiques du Bronze final et du premier âge du Fer de Corse

\begin{tabular}{|c|c|c|c|}
\cline { 2 - 4 } \multicolumn{1}{c|}{} & A.C.C. & Nusciaresa & Centre-Nord \\
\hline A.C.C. & & 0.34 & 0.16 \\
\hline Nusciaresa & 0.34 & & 0.21 \\
\hline Centre-Nord & 0.16 & 0.21 & \\
\hline
\end{tabular}

Figure 13 : degré de superposition typologique des ensembles céramiques des cinq phases articulées entre le Bronze final et le premier âge du Fer dans le sud de la Corse, selon l'indexation de Jaccard.

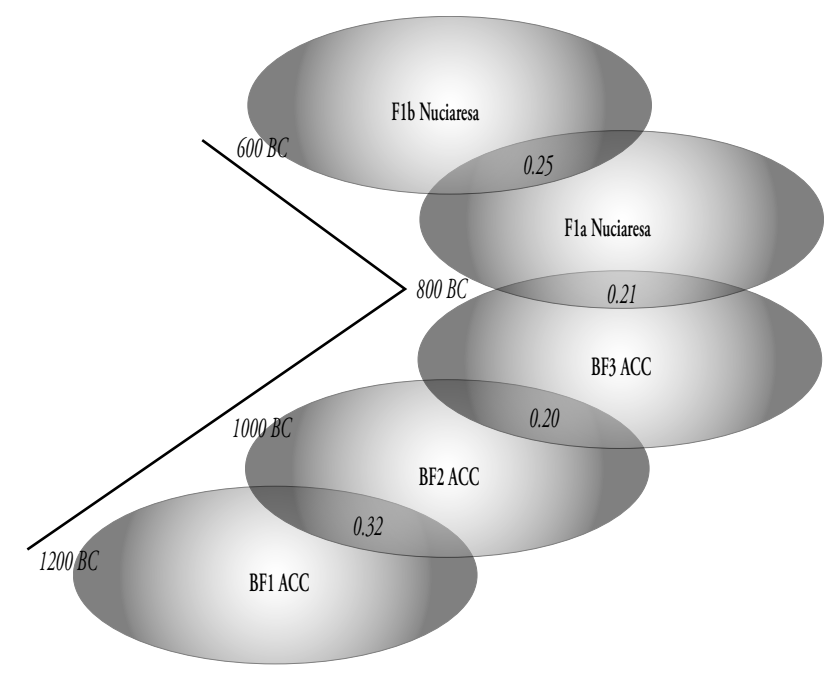


Figure 14 : habitation 1 de Cuciurpula en cours de fouille, premier âge du Fer. (DAO Laurent Bergerot, UMR MAP).

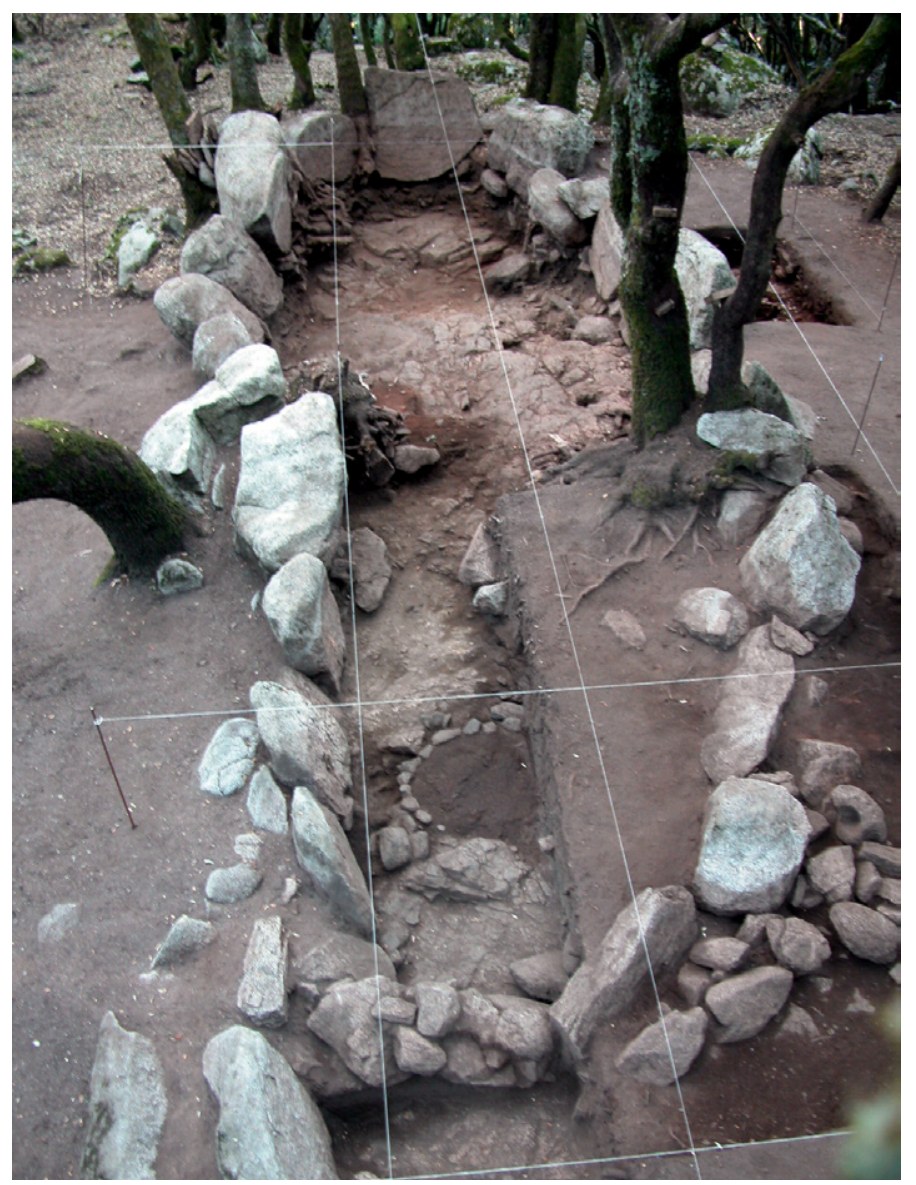

Les formes habitatives typiques des faciès méridionaux du Bronze final et du premier âge du Fer (fig. 14) incluent systématiquement des maisons ovalaires allongées aux soubassements faits de gros blocs à face plane disposée en parement interne [21]. Cette rémanence présente la particularité de s'exercer sur un espace tout à fait superposable (fig. 15 et 16). Plus au nord, sur la côte occidentale, plus particulièrement dans la vallée du Tàravu (sites de Valpuli et de Stilbè), l'architecture présente des formes proches mais des techniques privilégiant la pierre sèche, à la manière des navetes des Baléares du premier âge du Fer [22], sans toutefois qu'un lien direct entre les deux contextes insulaires ait pu être documenté jusqu'à aujourd'hui.

La précédente remarque sur les sphères productives méridionales qui illustreraient une certaine tendance à l'autarcie, doit ici être pondérée puisqu'on a pu individualiser quelques objets « exotiques », comme deux perles en verre syropalestinien provenant d'un contexte sépulcral (Cuciurpula, abri 2) [23], permettant pour la première fois dans I'île d'évoquer le vecteur phénicien, autour des $\mathrm{VII}^{\mathrm{e}}-\mathrm{VII}^{\mathrm{e}}$ siècles avant J.-C., soit une chronologie voisine de celle obtenue en Sardaigne pour des cas de figures superposables [24].

\footnotetext{
[21] LANFRANCHI et al. à paraître.

[22] Fornés et al. 2009 ; Garcia Amengual 2010.

[23] Milletti et al. 2012.

[24] Hayne 2010, 2012 ; Santocchini Gerg 2011 ;

SANTOCCHINI GERG, à paraître.
}

Figure 15 : localisation des sites à céramique « Apazzu-Castidetta-Cucuruzzu » et à céramique Nuciaresa.

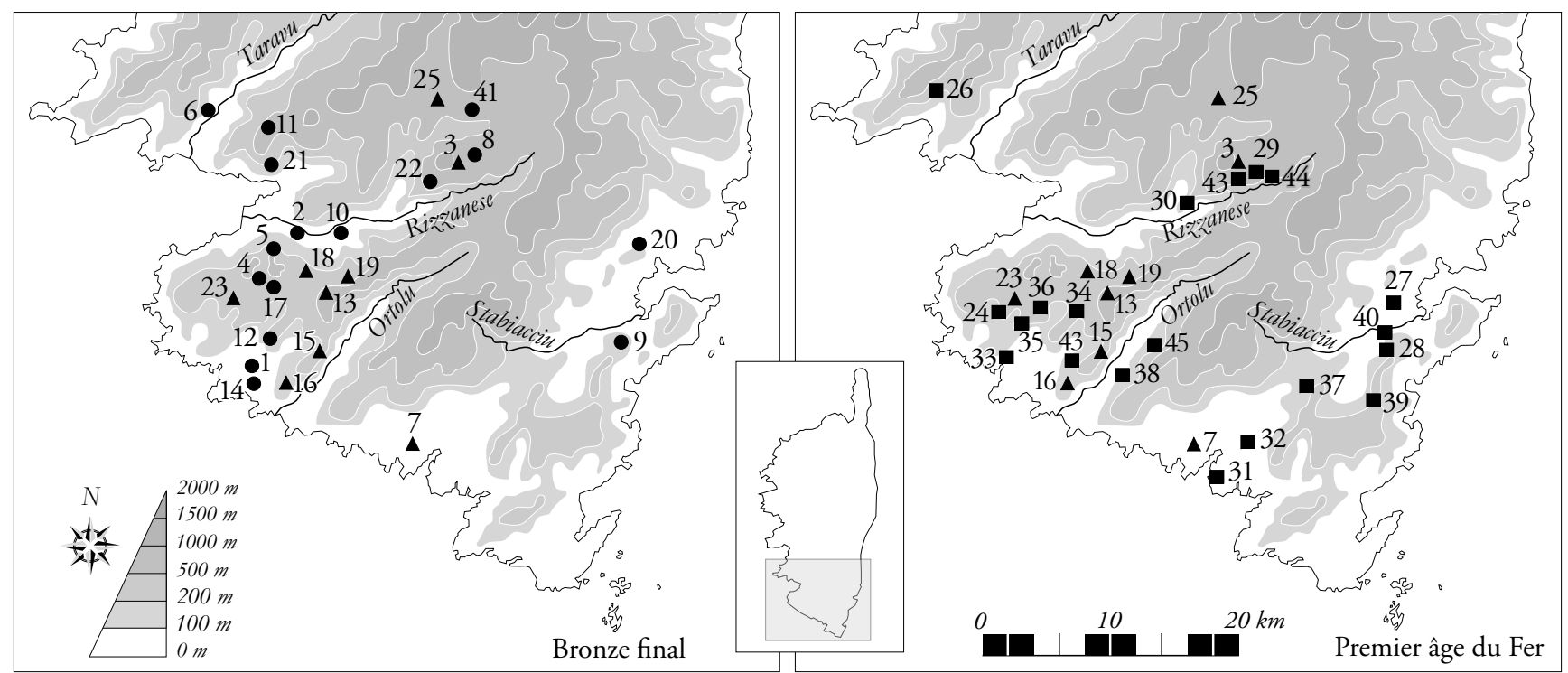

\section{- : Bronze final}

1 - Apazzu Vecchju
2 - Castidetta-Pozzone
3 - Cucuruzzu
4 - Alo-Bisughjè
5 - Baresi
6 - Basì
7 - Caldarellu
8 - Capula
9 - Ceccia

A : Bronze final et premier âge du Fer

19 - Torracone
20 - Torre
21 - Turricciola
22 - Tusiu
23 - Valchiria
24 - Canava
25 - Cuciurpula
26 - I Sapari
27 - Cozza Torta

\begin{tabular}{|c|c|}
\hline 28 - Tappa 2 & 37 - Cavaddu Biancu \\
\hline 29 - Nuciaresa-Saparaccia & 38 - Ranfonu \\
\hline 30 - Cumpulaghja & 39 - San Petru \\
\hline 31 - Ventilegne & 40 - Cileca \\
\hline 32 - Buffua & 41 - Puzzonu \\
\hline 33 - Magazene & 42 - Middari \\
\hline 34 - Acciola & 43 - Riccu \\
\hline 35-A Viccia & $44-$ Buri \\
\hline 36 - A Cota & 45 - Briccu Rossu \\
\hline
\end{tabular}


Figure 16 : répartition des habitats incluant des maisons de forme elliptique à soubassement monumental dans le sud de la Corse.

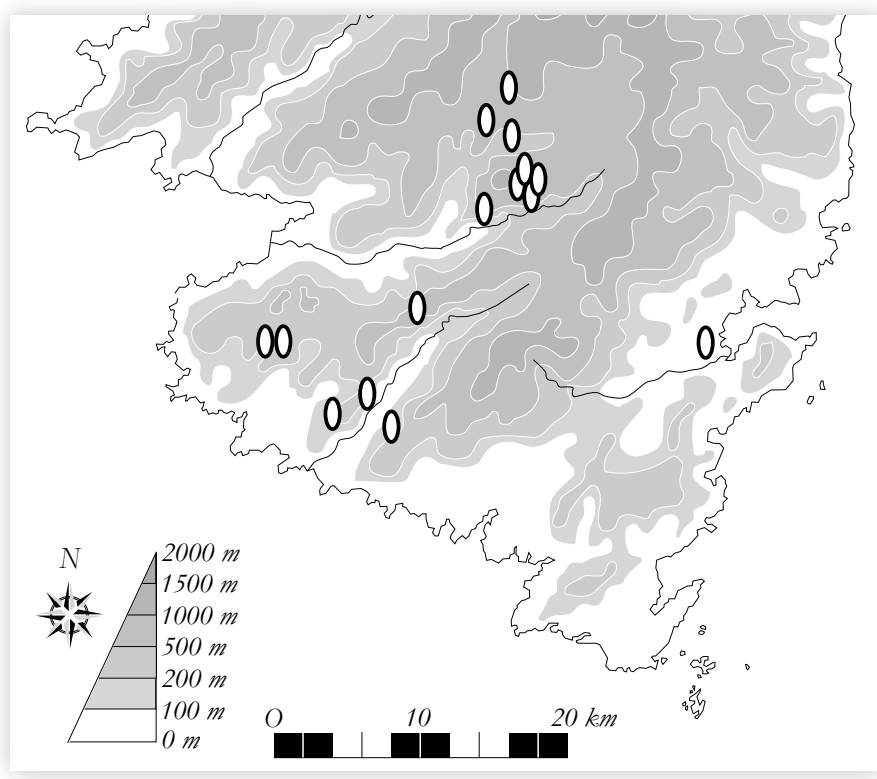

[25] Peche-Quilichini 2010.

[26] Antona et al. 2010.

[27] Peche-Quilichini \& Piccardi à paraître.
Un autre cas avéré d'importation est l'arrivée dans le sud de nucleus d'hématite, matériau utilisé par les autochtones comme colorant rouge à destination indéterminée. Ce matériau était peut-être en partie apporté depuis l'île d'Elbe, donc depuis un contexte étrusque, tout au long du viI ${ }^{\mathrm{e}}$ siècle, avant un arrêt assez brutal vers le milieu du $\mathrm{VI}^{\mathrm{e}}$ siècle, ce qui est remarquable car la date de 565-563 av. J.-C. correspond à I'installation des Grecs phocéens à Alalia, sur I'unique route reliant le sud de la Corse à I'antique Aethalie ; on serait donc tenté d'y voir un lien de cause à effet. Autre exemple d'occurrence, I'existence en Corse méridionale [25] et en Sardaigne septentrionale [26] de vases à dispositif de préhension zoomorphe (fig. 17) déposés en des contextes à forte signification symbolique supposée (tombes et dépôts de fondation), aux $\mathrm{IX}^{\mathrm{e}}-\mathrm{VII}^{\mathrm{e}}$ siècles av. J.-C. La rémanence de la pratique illustre probablement et partiellement des structures rituelles, voire mythologiques, communes à ces deux espaces.

Le second âge du Fer est marqué par une nouveauté : pour la première fois, la Corse exporte ses productions et ses modèles de vaisselle à pâte amiantée et à surfaçages au peigne vers l'archipel toscan et les cités étrusques nouvellement conquises par les Romains [27].

Figure 18 : géographie des parlers corses.

Figure 17 : panel de vases portant une anse en forme de protomé cornu, sud de la Corse, premier âge du Fer.

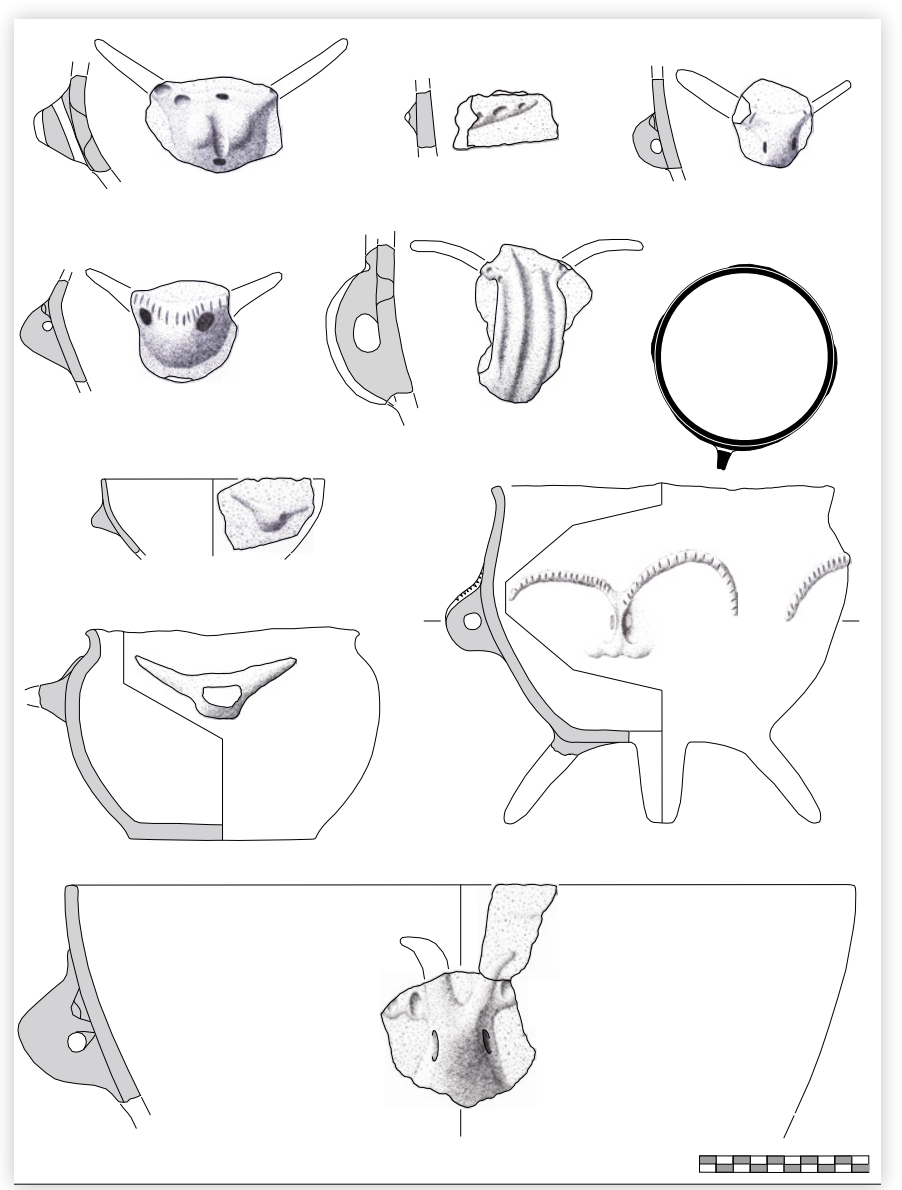

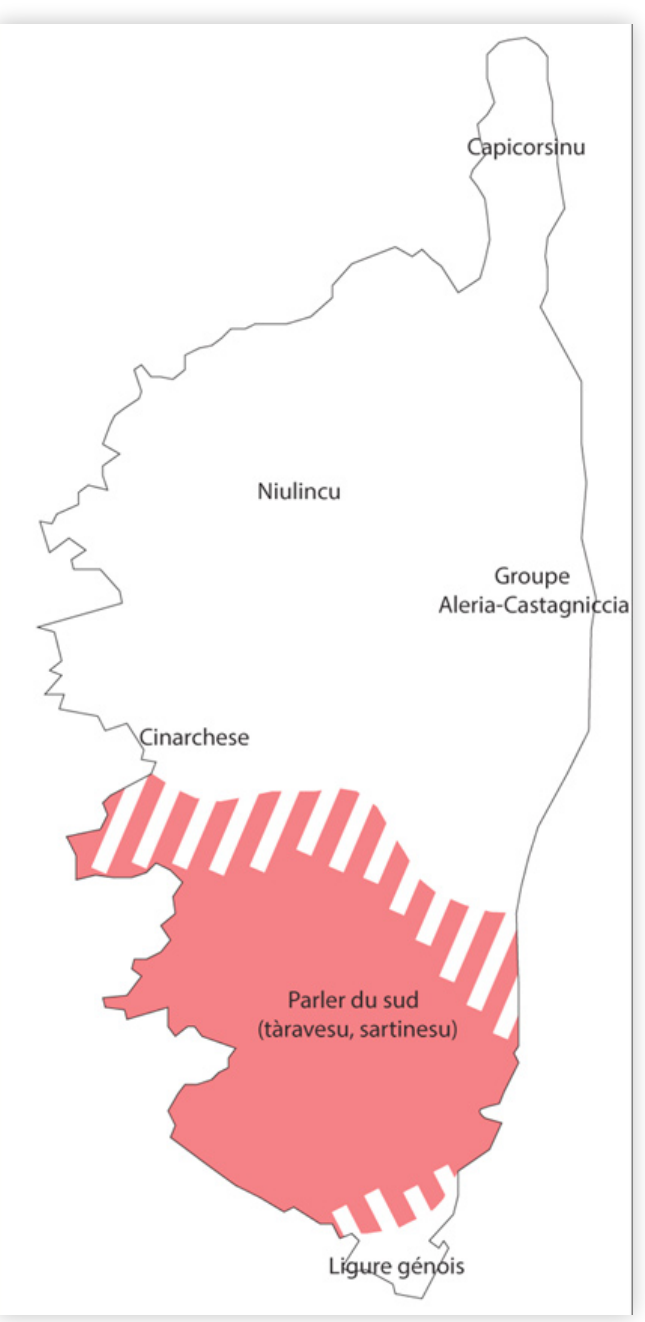


Des postulats véhiculés par les sources écrites, mais non démontrés, y voient le reflet matériel du commerce (ou du tribut ?) du miel insulaire, réputé durant l'Antiquité malgré une amertume devenue proverbiale (« Donner du miel corse aux abeilles du Mont Hybla » : offrir un cadeau ridicule à qui est digne de grands honneurs).

\section{UN TERRITOIRE MARGINAL, DIVISÉ ET OUVERT}

Pour conclure, à l'échelle de lîle, on remarquera surtout, époque après époque, l'amplification d'une distinction des secteurs méridionaux, qui tient probablement de l'expression d'une tradition mais qui s'explique aussi peut-être par des logiques géographiques et culturelles. Dans cet espace, la Sardaigne, pôle culturel dynamique, est visible depuis tous les sommets et franchir le détroit n'a jamais constitué une difficulté insurmontable. Comme on l'a vu, I'originalité des zones méridionales s'explique en partie par une influence sarde sur les productions matérielles et probablement sur un grand nombre d'éléments socio-économiques. Il convient toutefois de garder à l'esprit la possibilité du développement de faciès en réaction à l'impact sarde, par rebond et proportionnel à l'infiltration culturelle exogène. On notera en complément que la spécificité du secteur ne s'exerce pas qu'en termes archéologiques puisqu'on peut la vérifier encore aujourd'hui en linguistique (fig. 18) et en génétique [28]. Dans les vallées septentrionales, moins documentées, les contextes de l'âge du Fer traduisent des contacts plus affirmés avec les groupes de l'Italie tyrrhénienne.
Dans tous les cas, les mécanismes d'emprunt ne s'expriment que dans certaines niches productives, jamais selon une trame globalisante. Ce phénomène pourrait trouver des explications variées et combinées, à chercher dans le particularisme des processus de contact, dans le degré de réceptivité des groupes indigènes et dans bien d'autres éléments dont la complexité à quantifier et définir est propre aux champs d'investigation qui caractérisent les sciences humaines. Parmi les facteurs probables de développement des emprunts techno-stylistiques figure en bonne place I'existence d'une route de cabotage reliant l'Étrurie à la Sardaigne et dont la Corse profite indirectement grâce à sa position géographique depuis au moins la fin de l'âge du Bronze et probablement même avant. La Corse apparaît davantage comme un pôle récepteur qu'émetteur, en position de marge horizontale (territoire insulaire) et verticale (territoire montagneux) face à des sociétés toujours plus structurées et unitaires qui offriront un substrat solide aux civilisations antiques.

Inversement, la Corse est caractérisée par une tendance croissante à la régionalisation des faciès archéologiques tout au long de la Protohistoire. En transposant ces phénomènes sur le plan ethnique tel qu'il nous est décrit par les sources antiques, on pourrait être tenté de réaliser des rapprochements avec certaines descriptions, comme par exemple celle de Ptolémée d'Alexandrie au II ${ }^{\mathrm{e}}$ siècle ap. J.-C., dont le texte renvoie l'image d'une société morcelée et d'une île « comme une centaine au milieu d'une seule » (Isula, Centu ind'una sola, Canta u Populu Corsu, Isula Strana).

[28] Giovannini et al. 2006.

\section{BIBLIOGRAPHIE}

Antona, A. et al., 2010, « Spazi di lavoro e attività produttive nel villaggio nuragico La Prisgiona in località Capichera (Arzachena) », dans M. Milanese, P. Ruggeri \& C. Vismara (éd.), L'Africa romana. Atti del xvIII Convegno di studio (Olbia, décembre 2008), Roma, p. $1713-1734$.

Atzeni, E., 1966, «L'abri sous roche D' du village préhistorique de Filitosa », dans Actes du Congrès Préhistorique de France. Compte rendu de la XVIII ${ }^{e}$ session (Ajaccio, 1966), Paris, p. 169-192.

Atzeni, E. \& Depalmas, A., 2006, « Materiali "appenninici" da Filitosa », dans Istituto Italiano di Preistoria e Protostoria (éd.), Atti della XXXIX Riunione Scientifica dell'Istituto Italiano di Preistoria e Protostoria: materie prime e scambi nella Preistoria italiana (Florence, novembre 2004), Firenze, p. 1173-1184.

D'Anna, A. et al., 2006, « Espaces, territoires et mégalithes : le plateau de Cauria (Sartène, Corse-du-Sud) au Néolithique et à l'âge du Bronze », dans P. Duhamel (dir.), Impacts interculturels au Néolithique moyen : du terroir au territoire : sociétés et espaces. Actes du 25e Colloque interrégional sur le Néolithique (Dijon, octobre 2001), Revue Archéologique de l'Est suppl. 25, p. 191-213.

D'AnNA, A. et al., 2007, « Le plateau de Cauria à l'âge du Bronze : de la lecture événementielle à l'approche pluridisciplinaire anthropologique, hommage à Roger Grosjean », dans J. Evin (dir.), Un siècle de construction du discours scientifique en Préhistoire. $X X V I^{e}$ Congrès Préhistorique de France «Congrès du Centenaire » (Avignon, septembre 2004), Société Préhistorique Française, Paris, p. 331-346.

Fornés, J. et al., 2009, « «Más que una casa. Los navetiformes en el Bronce Balear»», dans Collectif (éd.), L'espai domèstic $i$ l'organització de la societat a la protohistòria de la Mediterrània occidental ( $I^{\text {er }}$ milleni aC). IV reunió internacional d'arqueologia de Calafell (6 al 9 de març de 2007), Calafell, p. 325-332. 
Garcia Amengual, E., 2010, « L'estat de les estructures navetiformes mallorquines », Mayurqa 33, p. 47-61.

Giovannoni, R. et al., 2012, «Structure génétique de la population corse », Anthropo 11, p. 37-50.

GosselaIn, O. P., 2002, Poteries du Cameroun méridional : styles techniques et rapports à l'identité (Monographies du CRA 26), Paris.

Hayne, J., 2010, « Entangled identities on Iron Age Sardinia ? », dans P. Van Dommelen \& A. B. Knapp (éd.), Material connections in the Ancient Mediterranean: mobility, materiality and mediterranean identities, London, p. 147-169.

HAYNe, J., 2012, Culture contact and exchange in Iron Age north Sardinia (900-200 BC), Thèse de doctorat, University of Glasgow.

LACHenAL, T., 2008, «Relations transalpines à l'âge du Bronze : état des données pour la Provence », dans D. Binder, X. Delestre \& P. Pergola (éd.), Archéologies transfrontalières : Alpes du sud, Côte d'Azur, Piémont et Ligurie : bilan et perspectives de recherche. Actes du colloque de Nice (Nice, décembre 2007), Monaco, p. 81-93.

Lachenal, T. \& Peche-Quilichini, K., 2009, «Relazioni culturali nel Mediterraneo nord-occidentale durante il Bronzo medio. Contributo delle tipologie ceramiche », dans M. G. Melis (dir.), Uomo e Territorio. Dinamiche di frequentazione e di sfruttamento delle risorse naturali nell'Antichità. Atti del Convegno Nazionale dei Giovanni Archeologi (Sassari, septembre 2006), Muros, p. 141-148.

LANFranchI, F. DE et al., à paraître, « Nouvelles données sur l'habitat du premier âge du Fer en Alta Rocca », dans J. de Lanfranchi (dir.), Quoi de neuf en archéologie ? Actes des XI Rencontres du Musée de l'Alta Rocca (Levie, novembre 2011).

Leandri, F. \& Peche-Quilichini, K., à paraître, « Iconographie comparée et contextualisée des statues-menhirs corses et des bronzetti anthropomorphes sardes », dans G. Rodriguez (dir.), Pierres levées du Néolithique à l'âge du Fer. Actes du $3^{e}$ colloque international sur la statuaire mégalithique (Saint-Pons-de-Thomières, septembre 2012).

Lechenault, M., 2011, Les trafics dans les îles de Méditerranée centrale et occidentale au Premier âge du Fer : la Corse des échanges, Thèse de Doctorat, Université de Lyon II.

Millettr, M. et al., 2012, «Cuciurpula, Serra-di-Scopamena/Sorbollano (Corse-du-Sud) : nuovi dati su un insediamento protostorico corso (campagne 2008-2011) », Materiali per Populonia 9-10, p. 377-444.

Peche-Quilichini, K., 2009a, « Fonds céramiques et vannerie dans le sud de la Corse au Bronze final », Bulletin de la Société Préhistorique Française 106, p. 569-580.

Peche-Quilichini, K., 2009b, «Révision chrono-culturelle des vaisselles de l'âge du Bronze de Filitosa-Turrichju (Sollacaro, Corse-du-Sud) », Documents d'Archéologie Méridionale 32, p. 161-210.

Peche-Quilichini, K., 2010a, «La ceramica del Bronzo finale nel sud della Corsica (sec. xil-Ix): la facies "Apazzu-CastidettaCucuruzzu" », dans N. Negroni Catacchio (éd.), Paesaggi reali e paesaggi mentali. L'alba dell'Etruria. Fenomeni di trasformazione e di continuità nei secoli XII-VIII. Atti del Nono Incontro di studi del comitato "Preistoria e Protostoria dell'Etruria" (Valentano et Pitigliano, septembre 2008), Milano, p. 573-593.

Peche-Quilichini, K., 2010b, « Le vase de fondation zoomorphe du premier âge du Fer de Cuciurpula (Serra-di-Scopamène/ Sorbollano, Corse-du-Sud) », Bulletin de la Société Préhistorique Française 107, p. 371-381.

Peche-Quilichini, K., 2011, « Les monuments turriformes de l'âge du Bronze en Corse : tentative de caractérisation spatiale et chronologique sur fond d'historiographie », dans D. Garcia (dir.), L'âge du Bronze en Méditerranée. Recherches récentes. Séminaire d'Antiquités nationales et de Protohistoire européenne d'Aix-en-Provence, Paris, p. 155-169.

Peche-Quilichini, K., à paraître 1, « Bols, paniers et grains de riz ». Les vaisselles céramiques du Bronze final et du premier âge du Fer de Corse. Façonnage, morphologie, modes de consommation, chronologie et significations culturelles d'une production insulaire (Monographies d'Archéologie Méditerranéenne), Montpellier-Lattes.

Peche-Quilichini, K., à paraître 2, «Chronologie, productions matérielles et dynamiques socio-culturelles : le point sur le séquençage de l'âge du Bronze de la Corse », dans J. de Lanfranchi (dir.), Quoi de neuf en archéologie ? Actes des xI Rencontres du Musée de l'Alta Rocca (Levie, novembre 2011).

Peche-Quilichini, K., à paraître 3, « Note sur deux tasses à carène basse et leur intégration dans les ensembles céramiques du Bronze ancien corse », Bulletin de la Société des Sciences Historiques et Naturelles de la Corse.

Peche-Quilichini, K., à paraître 4, «Influences, inspirations ou transferts? La question des affinités corso-toscanes dans les productions matérielles protohistoriques », dans G. Camporeale \& D. Briquel (dir.), Aleria e Populonia. Atti del xxviII Convegno di Studi Etruschi ed Italici (Bastia, Piombino, octobre 2011).

Peche-Quilichini, K. \& Piccardi, E., à paraître, «Domestic uses, trading and afterwards functions of pottery in the $2^{\text {nd }}$ Iron Age northern Tyrrhenian sea: potential identity evidences », dans Collectif (éd.), Identity and connectivity. Proceedings of the $x v I^{\text {th }}$ Symposium on Mediterranean Archaeology (Florence, mars 2012) (B.A.R. International Series).

Peche-Quilichini, K. et al., à paraître, « Les matrices de fusion protohistoriques de Corse : état de la recherche et découvertes récentes », dans T. Perrin, I. Senepart, F. Leandri \& J. Cauliez (dir.), Chronologie de la Préhistoire Récente dans le Sud de la France. Acquis 1992-2012. Actualités de la recherche. Actes des $x$ e Rencontres Méridionales de Préhistoire Récente (Ajaccio, octobre 2012).

Roth, A. \& Congès, G., 1976, « Un dépotoir protohistorique près de l'étang de Diane », Archeologia Corsa 1, p. $97-112$.

Santocchini Gerg, S., 2011, Incontri tirrenici: le relazioni fra Fenici, Sardi ed Etruschi in Sardegna (630-480 a.C.), Thèse de doctorat, Università degli Studi di Sassari.

Santocchini Gerg, S., à paraître, «Le importazioni etrusche nella Sardegna d'età arcaica: nuovi dati e spunti di riflessione », dans A. Bastia (dir.), vI e Congrès des Études Phéniciennes et Puniques (Tunis, novembre 2009).

Sebis, S., 1995, « La ceramica nuragica del Bronzo medio (XVI-XIV sec.) e del Bronzo recente (XIII-XII sec.) nell'Oristanese », dans Collectif (éd.), La ceramica artistica, d'uso e da costruzione nell'Oristanese dal Neolitico ai giorni nostri. Atti del Primo Convegno "La ceramica raconta la Storia" (Oristano-Cabras, octobre 1993), Oristano, p. 137-156.

VITAL, J., 1998, « À propos des rapports culturels entre I'Italie nord-occidentale et le sud-est de la France du Bronze moyen au Bronze final », dans Istituto Italiano di Preistoria e Protostoria (éd.), Preistoria e Protostoria del Piemonte. Atti della xxxII Riunione Scientifica dell'Istituto Italiano di Preistoria e Protostoria (Alba, septembre-octobre 1995), Firenze, p. 473-481. 